\title{
CONF-980672--
}

\section{SHELL-MODEL MONTE CARLO STUDIES OF NUCLEI FAR FROM STABILITY}

\author{
D. J. Dean
}

Physics Division, Oak Ridge National Laboratory, Oak Ridge, TN and

Department of Physics, University of Tennessee, Knoxville, TN

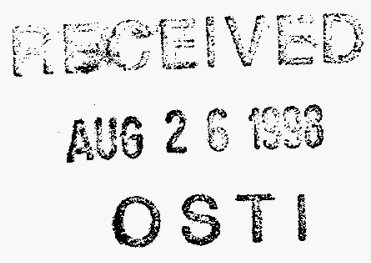

Proceedings of 2nd International Conference on Exotic Nuclei and Atomic Masses (ENAM98)

Bellaire, Michigan

June 23-27, 1998

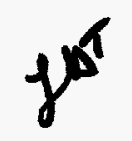

*Oak Ridge National Laboratory is managed by Lockheed Martin Energy Research Corp. for the U.S. Department of Energy under Contract No. DE-AC05-96OR22464.

"The submitted manuscript has been authored by a contractor of the U.S. Government under contract No. DE-AC05-96OR22464. Accordingly, the U.S. Government retains a nonexclusive, royalty-free license to publish or reproduce the published form of this contribution, or allow others to do so, for U.S. Government purpases." 


\section{DISCLAIMER}

This report was prepared as an account of work sponsored by an agency of the United States Government. Neither the United States Government nor any agency thereof, nor any of their employees, make any warranty, express or implied, or assumes any legal liability or responsibility for the accuracy, completeness, or usefulness of any information, apparatus, product, or process disclosed, or represents that its use would not infringe privately owned rights. Reference herein to any specific commercial product, process, or service by trade name, trademark, manufacturer, or otherwise does not necessarily constitute or imply its endorsement, recommendation, or favoring by the United States Government or any agency thereof. The views and opinions of authors expressed herein do not necessarily state or reflect those of the United States Government or any agency thereof. 


\section{DISCLAIMER}

Portions of this document may be illegible in electronic image products. Images are produced from the best available original document. 


\title{
Shell-Model Monte Carlo Studies of Nuclei Far From Stability
}

\author{
David J. Dean \\ Physics Division, Oak Ridge National Laboratory, Oak Ridge, TN \\ and Physics Department, University of Tennessee, Knoxville, TN ${ }^{1}$
}

\begin{abstract}
The shell-model Monte Carlo (SMMC) technique transforms the traditional nuclear shell-model problem into a path-integral over auxiliary fields. I describe below the method and its applications to two physics issues: calculations of electron-capture rates, and exploration of pairing correlations in unstable nuclei.
\end{abstract}

Studies of nuclei far from stability have long been a goal of nuclear science. Nuclei on either side of the stability region, either neutron-rich or deficient, are being produced at new radioactive beam facilities across the world. At these facilities, and with the help of advances in nuclear many-body theory, the community will address many of the key physics issues, including: mapping of the neutron and proton drip lines, thus exploring the limits of stability; understanding effects of the continuum on weakly bound nuclear systems; understanding the nature of shell gap melting in very neutron-rich systems; determining nuclear properties needed for astrophysics; investigating deformation, spin, and pairing properties of systems far from stability; and analyzing microscopically unusual shapes in unstable nuclei.

The range and diversity of nuclear behavior, as indicated in the above list of ongoing and planned experimental investigations, have naturally engendered a host of theoretical models. Short of a complete solution to the many-nucleon problem, the interacting shell model is widely regarded as the most broadly capable description of low-energy nuclear structure, and the one most directly traceable to the fundamental many-body problem. Difficult though it may be, solving the shell-model problem is of fundamental importance to our understanding of the correlations found in nuclei.

My research over the past few years has been in the area of the nuclear shell model solved not by diagonalization, but by integration. In what follows, I will describe the shell-model Monte Carlo (SMMC) method, and discuss two recent and interesting

1) Oak Ridge National Laboratory (ORNL) is managed by Lockheed Martin Energy Research Corp. for the U.S. Department of Energy under contract number DE-AC05-96OR22464. This work was supported in part through grant DE-FG02-96ER40963 from the U.S. Department of Energy. 
results obtained from theory. These include calculations of electron-capture rates in $f p$-shell nuclei, and pairing correlations in medium-mass nuclei near $\mathrm{N}=\mathrm{Z}$.

Investigations of both ground state and thermal properties of nuclei have been described using the SMMC technique [1]. This method offers an alternative way to calculate nuclear structure properties, and is complementary to direct diagonalization. SMMC cannot find, nor is it designed to find, every energy eigenvalue of the Hamiltonian. It is designed to give thermal or ground-state expectation values for various one- and two-body operators. Indeed, for larger nuclei, SMMC may be the only way to obtain information on the thermal properties of the system from a shell-model perspective. The partition function of the imaginary-time many-body propagator, $U=\exp (-\beta \hat{H})$ where $\beta=1 / T$ and $T$ is the temperature of the system in $\mathrm{MeV}$, is used to calculate the expectation values of any observable $\hat{\Omega}$ with

$$
\langle\hat{\Omega}\rangle=\frac{\operatorname{Tr} \hat{U} \hat{\Omega}}{\operatorname{Tr} \hat{U}}
$$

Since $\hat{H}$ contains many terms that do not commute, one must discretize $\beta=N_{t} \Delta \beta$. Finally, two-body terms in $\hat{H}$ are linearized through the Hubbard-Stratonovich transformation, which introduces auxiliary fields over which one must integrate to obtain physical answers. The method can be summarized as

$$
\begin{aligned}
Z & =\operatorname{Tr} \hat{U}=\operatorname{Tr} \exp (-\beta \hat{H}) \rightarrow \operatorname{Tr}[\exp (-\Delta \beta \hat{H})]^{N_{t}} \\
& \rightarrow \int \mathcal{D}[\sigma] G(\sigma) \operatorname{Tr} \prod_{n=1}^{N_{t}} \exp \left[\Delta \beta \hat{h}\left(\sigma_{n}\right)\right]
\end{aligned}
$$

where $\sigma_{n}$ are the auxiliary fields (there is one $\sigma$-field for each two-body matrixelement in $\hat{H}$ when the two-body terms are recast in quadratic form), $\mathcal{D}[\sigma]$ is the measure of the integrand, $G(\sigma)$ is a Gaussian in $\sigma$, and $\hat{h}$ is a one-body Hamiltonian. Thus, the shell-model problem is transformed from the diagonalization of a large matrix to one of large dimensional quadrature. Dimensions of the integral can reach up to $10^{5}$ for rare-earth systems, and it is thus natural to use Metropolis random walk methods to sample the integrands. Such integration can most efficiently be performed on massively parallel computers.

The SMMC method is also used to calculate the response function $R_{\mathcal{A}}(\tau)$ of an operator $\mathcal{A}$ at an imaginary-time $\tau$. The operator $\mathcal{A}$ may be of the one-body form, $a^{\dagger} a$, as is the case with Gamow-Teller distributions, or of the pair creation (or annihilation) form, $a^{\dagger} a^{\dagger}$, as is the case for pair transfer calculations. The response describes the dynamical behavior of the nucleus under the influence of the operator, and is given by

$$
R_{\mathcal{A}}(\tau) \equiv\left\langle\hat{\mathcal{A}}^{\dagger}(\tau) \hat{\mathcal{A}}(0)\right\rangle=\frac{\operatorname{Tr}\left[e^{-(\beta-\tau) \hat{H}} \hat{\mathcal{A}}^{\dagger} e^{-\tau \hat{H}} \hat{\mathcal{A}}\right]}{\operatorname{Tr}\left[e^{-\beta \hat{H}}\right]}
$$

The strength distribution, $S_{\mathcal{A}}(E)$, is related to $R_{\mathcal{A}}(\tau)$ by a Laplace Transform, 


$$
R_{\mathcal{A}}(\tau)=\int_{-\infty}^{\infty} S_{\mathcal{A}}(E) e^{-\tau E} d E
$$

Note that $E$ is the energy transfer within the parent nucleus, and that the strength distribution $S_{\mathcal{A}}(E)$ has units of $\mathrm{MeV}^{-1}$.

The impact of nuclear structure on astrophysics has become increasingly important, particularly in the fascinating and presently unsolved problem of type-II supernovae explosions. One key ingredient of the precollapse scenario is the electron capture cross section on nuclei $[2,3]$. An important contribution to electron capture cross sections in supernovae environments is the Gamow-Teller (GT) strength distribution. This strength distribution, calculated in SMMC using Eqs. $(3,4)$ above, is used to find the energy-dependent cross section for electron capture. In order to obtain the electron capture rates, the cross section is then folded with the flux of a degenerate relativistic electron gas [4]. Note that the Gamow-Teller distribution is calculated at the finite nuclear temperature which, in principle, is the same as the one of the electron gas.

It is important to calculate the GT strength distributions reasonably accurately for both the total strength, and the position of the main GT peak in order to have a quantitative estimate for the electron capture rates. For astrophysical purposes, calculating the rates to within a factor of two is required. I concentrate here on mid- $f p$ shell results for the electron capture cross sections [4]. The Kuo-Brown interaction [5], modified in the monopole terms by Zuker and Poves [6], was used throughout these $p f$-shell calculations. This interaction reproduces quite nicely the ground- and excited-state properties of mid- $f p$ shell nuclei [7,8], including the total Gamow-Teller strengths and distributions, where the general overall agreement between theory and experiment [9] is quite reasonable. The SMMC technique allows one to probe the complete $0 \hbar \omega f p$-shell region, without any parameter adjustments to the Hamiltonian, although the Gamow-Teller operator has been renormalized by the standard factor of 0.8 .

Do the electron capture rates presented here indicate potential implications for the pre-collapse evolution of a type II supernova? To make a judgement on this important question, one should compare in Table I the SMMC rates for selected nuclei with those currently used in collapse calculations [3]. For the comparison, I choose the same physical conditions as assumed in Tables 4-6 in [3]. Table I also lists the partial electron capture rate which has been attributed to Gamow-Teller transitions in Ref. [3]. Note that for even parent nuclei, the present rate approximately agrees with the currently recommended total rate. A closer inspection, however, shows significant differences between the present rate and the one attributed to the Gamow-Teller transition in [3]. The origin of this discrepancy is due to the fact that Ref. [10] places the Gamow-Teller resonance for even-even nuclei systematically at too high an excitation energy. This shortcoming has been corrected for in Refs. $[10,3]$ by adding an experimentally known low-lying strength in addition to the one attributed to Gamow-Teller transitions. However, the overall good agreement between the SMMC results for even-even nuclei and the recommended rates indicates 
TABLE 1. Comparisons of the SMMC electron capture rates with the total $\left(\lambda_{\mathrm{ec}}\right)$ and partial Gamow-Teller $\left(\lambda_{\mathrm{ec}}^{\mathrm{GT}}\right)$ rates as given in Ref. [3]. Physical conditions at which the comparisons were made are given in the last column.

\begin{tabular}{|c|c|c|c|c|}
\hline Nucleus & $\begin{array}{c}\lambda_{\mathrm{ec}}\left(\mathrm{sec}^{-1}\right) \\
(\text { SMMC) }\end{array}$ & $\begin{array}{c}\lambda_{\mathrm{ec}}\left(\mathrm{sec}^{-1}\right) \\
\text { (Ref. [3]) }\end{array}$ & $\begin{array}{c}\lambda_{\text {ec }}^{G T}\left(\mathrm{sec}^{-1}\right) \\
\text { (Ref. [3] })\end{array}$ & Conditions \\
\hline${ }^{55} \mathrm{Co}$ & $3.89 \mathrm{E}-04$ & $1.41 \mathrm{E}-01$ & $1.23 \mathrm{E}-01$ & $\rho_{7}=5.86, T_{9}=3.40, Y_{e}=0.47$ \\
${ }^{57} \mathrm{Co}$ & $3.34 \mathrm{E}-06$ & $3.50 \mathrm{E}-03$ & $1.31 \mathrm{E}-04$ & $\rho_{7}=5.86, T_{9}=3.40, Y_{e}=0.47$ \\
${ }^{54} \mathrm{Fe}$ & $7.83 \mathrm{E}-05$ & $3.11 \mathrm{E}-04$ & $9.54 \mathrm{E}-07$ & $\rho_{7}=5.86, T_{9}=3.40, Y_{e}=0.47$ \\
${ }^{55} \mathrm{Fe}$ & $1.20 \mathrm{E}-08$ & $1.61 \mathrm{E}-03$ & $1.16 \mathrm{E}-07$ & $\rho_{7}=5.86, T_{9}=3.40, Y_{e}=0.47$ \\
${ }^{56} \mathrm{Ni}$ & $3.47 \mathrm{E}-02$ & $1.60 \mathrm{E}-02$ & $6.34 \mathrm{E}-03$ & $\rho_{7}=5.86, T_{9}=3.40, Y_{e}=0.47$ \\
${ }^{58} \mathrm{Ni}$ & $1.01 \mathrm{E}-03$ & $6.36 \mathrm{E}-04$ & $4.04 \mathrm{E}-06$ & $\rho_{7}=5.86, T_{9}=3.40, Y_{e}=0.47$ \\
${ }^{60} \mathrm{Ni}$ & $7.39 \mathrm{E}-05$ & $1.49 \mathrm{E}-06$ & $4.86 \mathrm{E}-07$ & $\rho_{7}=5.86, T_{9}=3.40, Y_{e}=0.47$ \\
\hline${ }^{59} \mathrm{Co}$ & $3.44 \mathrm{E}-07$ & $2.09 \mathrm{E}-04$ & $6.37 \mathrm{E}-05$ & $\rho_{7}=10.7, T_{9}=3.65, Y_{e}=0.455$ \\
${ }^{57} \mathrm{Co}$ & $2.06 \mathrm{E}-05$ & $7.65 \mathrm{E}-03$ & $3.69 \mathrm{E}-04$ & $\rho_{7}=10.7, T_{9}=3.65, Y_{e}=0.455$ \\
${ }^{55} \mathrm{Fe}$ & $1.07 \mathrm{E}-07$ & $3.80 \mathrm{E}-03$ & $5.51 \mathrm{E}-07$ & $\rho_{7}=10.7, T_{9}=3.65, Y_{e}=0.455$ \\
${ }^{56} \mathrm{Fe}$ & $9.80 \mathrm{E}-06$ & $4.68 \mathrm{E}-07$ & $6.60 \mathrm{E}-10$ & $\rho_{7}=10.7, T_{9}=3.65, Y_{e}=0.455$ \\
${ }^{54} \mathrm{Fe}$ & $3.84 \mathrm{E}-04$ & $9.50 \mathrm{E}-04$ & $3.85 \mathrm{E}-06$ & $\rho_{7}=10.7, T_{9}=3.65, Y_{e}=0.455$ \\
${ }^{51} \mathrm{~V}$ & $1.06 \mathrm{E}-06$ & $1.24 \mathrm{E}-05$ & $9.46 \mathrm{E}-09$ & $\rho_{7}=10.7, T_{9}=3.65, Y_{e}=0.455$ \\
${ }^{52} \mathrm{Cr}$ & $1.32 \mathrm{E}-04$ & $2.01 \mathrm{E}-07$ & $1.59 \mathrm{E}-10$ & $\rho_{7}=10.7, T_{9}=3.65, Y_{e}=0.455$ \\
${ }^{60} \mathrm{Ni}$ & $3.61 \mathrm{E}-04$ & $7.64 \mathrm{E}-06$ & $2.12 \mathrm{E}-06$ & $\rho_{7}=10.7, T_{9}=3.65, Y_{e}=0.455$ \\
\hline
\end{tabular}

that the SMMC approach also accounts correctly for this low-lying strength. This has already been deduced from the good agreement between SMMC Gamow-Teller distributions and data including the low-energy regime [9]. Thus, for even-even nuclei, the SMMC approach is able to predict the total electron capture rate rather reliably, even if no experimental data are available. Note that the SMMC rate is somewhat larger than the recommended rate for ${ }^{56} \mathrm{Fe}$ and ${ }^{60} \mathrm{Ni}$. In both cases the experimental Gamow-Teller distribution is known and agrees well with the SMMC results [9]. While the proposed increase of the rate for ${ }^{60} \mathrm{Ni}$ is not expected to have noticeable influence on the pre-collapse evolution, the increased rate for ${ }^{56} \mathrm{Fe}$ makes this nucleus an important contributor in the change of $Y_{e}$ during the collapse (see Table 15 of [3]).

For electron capture on odd- $A$ nuclei, observe that the SMMC rates, derived from the Gamow-Teller distributions, are significantly smaller than the recommended total rate. This is due to the fact that for odd- $A$ nuclei the Gamow-Teller transition peaks at rather high excitation energies in the daughter nucleus. The electron capture rate on odd- $A$ nuclei is therefore carried by weak transitions at low excitation energies. Comparing the SMMC rates to those attributed to Gamow-Teller transitions in Refs. [10,3] reveals that the latter have been, in general, significantly overestimated which is caused by the fact that the position of the Gamow-Teller resonance is usually put at too low excitation energies in the daughter. The SMMC calculation implies that the Gamow-Teller transitions should not contribute noticeably to the electron capture rates on odd- $A$ nuclei at the low temperatures studied in Tables 14-16 in [3]. Thus, the rates for odd- $A$ nuclei given in these tables should generally be replaced by the non-Gamow-Teller fraction. 


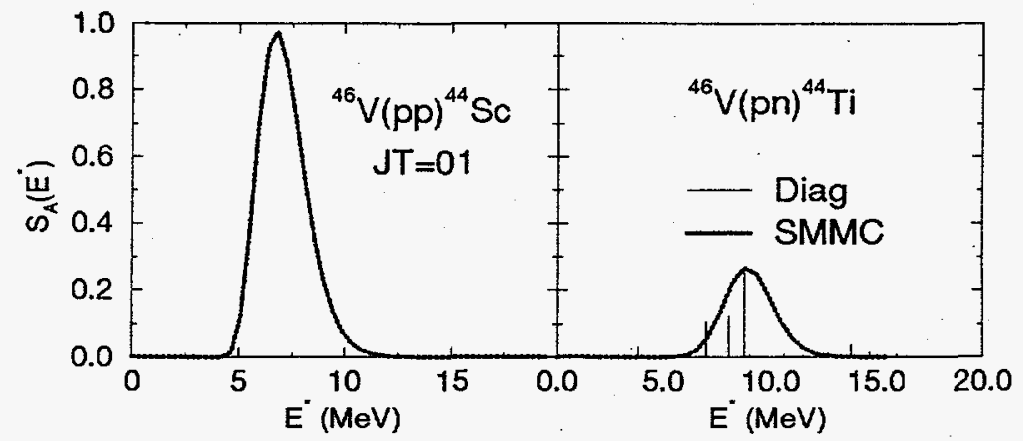

FIGURE 1. Left: proton pair strength distribution for ${ }^{46} \mathrm{~V}$ : Right: proton-neutron ( $\left.\mathrm{T}=1\right)$ pair strength distribution. SMMC: thick line; direct diagonalization: impulses.

I would now like to turn to the subject of pair correlations in nuclei, and calculations aimed at their understanding. Nuclei near $\mathrm{N}=\mathrm{Z}$ offer a unique place to study proton-neutron pairing, particularly in the isospin $\mathrm{T}=1$ channel. In fact, most heavy odd-odd, $\mathrm{N}=\mathrm{Z}$ nuclei beyond ${ }^{40} \mathrm{Ca}$ have total spin $\mathrm{J}=0, \mathrm{~T}=1$ ground states. Theoretical studies have shown that many of these nuclei have enhanced $\mathrm{T}=1$ proton-neutron correlations when compared to their even-even counterparts. These correlations are to a lesser extent present in even-even systems, but tend to decrease as one moves away from $\mathrm{N}=\mathrm{Z}$. In at least one nucleus in the mass 70 region, ${ }^{74} \mathrm{Rb}$, there is experimental evidence for a ground state $T=1$ band [11].

Experimentally, pair correlations can best be measured by pair transfer on nuclei. Although total cross sections are typically underpredicted when one employs spectroscopic factors computed from the shell model, relative two-nucleon spectroscopic factors within one nucleus are more reliable. Therefore, it is necessary for one to calculate and measure pair transfer from both the ground and excited states in a nucleus.

The SMMC method may be used to calculate the strength distribution of the pair annihilation operator $A_{J T T_{z}}$, as defined in [1]. The total strength of these pairing operators, i.e. the expectation $\left\langle A_{J T}^{\dagger} A_{J T}\right\rangle$, has been studied previously as a function of mass, temperature [12,13], and rotation [14]. I would like to briefly present here for the first time the strength distributions of the pair operators as calculated in SMMC. The strength distribution for the pair transfer spectroscopic factors is proportional to $\left\langle A-2\left|A_{J T}\right| A\right\rangle$, and is calculated by the inversion of Eq. (4).

In future work, I will discuss the strength distributions in detail. Here I would 
like to briefly conclude by demonstrating that the SMMC results and the direct diagonalization results agree very nicely for the proton pair strength distributions in the ground state of ${ }^{46} \mathrm{~V}$. This is demonstrated in the left panel of the figure. Shown in the right panel is the isovector proton-neutron pairing strength distribution with respect to the daughter nucleus. Notice that the overall strength is much larger in the proton-neutron channel, as discussed previously in [13], and that the peak is several $\mathrm{MeV}$ lower in excitation relative to the like-particle channel. In both cases the strength distribution in ${ }^{46} \mathrm{~V}$ differs significantly from that found in ${ }^{48} \mathrm{Cr}$, where one finds that the dominant component is a ground-state to ground-state transition involving mainly particles in the $0 f_{7 / 2}$ single-particle state. In both odd-odd $\mathrm{N}=\mathrm{Z}$ channels, the distribution is fairly highly fragmented.

In this proceedings, I have used two specific examples (there are several others) for which the SMMC calculations have proven very useful in understanding the properties of nuclei in systems where the number of valence particles prohibits the use of more traditional approaches. The method has proven to be a valuable tool to further our understanding of nuclear structure and astrophysics. While I have concentrated mainly on stable or proton-deficient nuclei in this contribution, continuing developments in the areas of multi-major shell calculations will, in the near future, provide much information on very neutron-rich nuclei.

\section{REFERENCES}

1. Koonin S.E., Dean D.J., and Langanke, K., Phys. Repts. 278, 1 (1997), and references therein.

2. Bethe H.A., Rev. Mod. Phys., 62, 801 (1990).

3. Aufderheide M.B., Fushiki I., Woosley S.E., Hartman D.H., Astrophys. J. Supp. 91, 389 (1994).

4. Dean D.J., Langanke K., Chatterjee L., Radha P.B., and Strayer M.R., Phys. Rev. C58, 536 (1998).

5. Kuo T. T. S. and Brown G. E., Nucl. Phys. A114, 241 (1968).

6. Poves A. and Zuker A., Phys. Repts. 70, 235 (1981).

7. Caurier E., Zuker A., Poves A., and Martinez-Pinedo G., Phys. Rev. C50, 225 (1994).

8. Langanke K., Dean D.J., Radha P.B., Alhassid Y., and Koonin S.E., Phys. Rev. C52, 718 (1995).

9. Radha P.B., Dean D.J., Koonin S.E., Langanke K., and Vogel P., Phys. Rev. C56, 3079 (1997).

10. Fuller G.M., Fowler W.A., and Newman M.J., Astrophys. J. 48, 279 (1982).

11. Rudolph D., et al, Phys. Rev. Lett. 76, 376 (1996).

12. Langanke K., Dean D.J., Radha P.B., and Koonin S.E., Nucl. Phys. A602, 244 (1996).

13. Langanke K., Dean D.J., Radha P.B., and Koonin S.E., Nucl. Phys. A613, 253 (1997).

14. Dean D.J., Koonin S.E., Langanke K., and Radha P.B., Phys. Lett. B399, 1 (1997) 